\title{
CHARACTERIZING CAVITATION EROSION PARTICLES BY ANALYSIS OF SEM IMAGES
}

\author{
Abouel-Kasem, A. \\ Mechanical Engineering Department, Faculty of Engineering, Assiut \\ University, Assiut (71516), Egypt \\ E-mail: abouelkasem@yahoo.com
}

(Received September 20, 2006 Accepted October 14, 2006)

\begin{abstract}
Detailed surface characteristics of the particles produced during cavitation erosion may well be significant in monitoring the cavitation erosion progress. Utilizing computer image analysis techniques, the size and shape factor for particles of Al-99.999 were analysed. The particle morphology features were also clarified. In incubation period, The particles have distinctive characteristics which differed from that for the subsequent periods. These characteristics include the value of longitudinal ratio and roundness factor, limit size range and morphological features such as lamella shape, folding, curving and one of the particle surfaces was the virgin surface. In acceleration, steady-rate and attenuation period, the particles have a wide size range and larger thickness compared with that for the incubation period. The maximum particle size was in acceleration and steady-rate period, and it was about $360 \mu \mathrm{m}$. For all the cavitation erosion rate periods, the particles were out of sphericity and they have a roundness factor larger than 2. The particle generation mechanism is fatigue failure.
\end{abstract}

KEYWORDS: Cavitation erosion, Particles, Image analysis, Erosion mechanisms, Al-99.999.

\section{1- INTRODUCTION}

Cavitation erosion has long been recognized as one of the major problems in the design and operation of modern high-speed flow systems. It is a serious problem in turbo machinery, engine combustion chambers, flow control valves, fast nuclear reactors, high speed mixing systems, high-frequency ultrasonic devices, etc. Therefore, a growing interest has been focused on such problem in order to find practical methods for predicting the damage development and the erosion rates precisely and for clarifying the erosion mechanism. Through such understanding cavitation erosion can be prevented, or at least its effects can be minimized in order to enhance the performance of such systems and increase their operating life. 
Wear particle analysis is a widespread method to characterize the tribological condition of mechanical systems [1]. For wear particle analysis, the most relevant features include size, shape, surface texture, edge detail, and thickness ratio [2]. The particle characteristics are sufficiently specific so that the operating wear modes within equipment may be determined, allowing prediction of the imminent behavior of the machine. Often action may be taken to correct the abnormal wear mode without overhaul, whilst a timely servicing can prevent costly secondary damage [3].

Kenkeramth and thiruvenqadam [5] analyzed the particles, and reported that many particles were quite irregular in shape. Also, they observed spherical particles among the cavitation erosion debris. Tomlinson and Chapman [6] examined the eroded particles of commercially pure copper and reported that the particles eroded by amplitude of 13.5 and $67.5 \mu \mathrm{m}$ had a similar size and shape. Ahmed et al. [7] observed both the eroded surface and removed particles by cavitation erosion of SUS 304 during the incubation period. They concluded that the dominant mode of cavitation erosion is fatigue failure. Moreover, they classified the gathered particles into three types according to their shapes, namely the longitudinal, the triangular and the mixed quadrangle hexagon. Also, they reported that the spherical particles, which were observed in a previous study [5] on cavitation erosion, were not observed. Hattori and Nakao [8] examined the removed particles for the different stages of erosion rate. Using the image analyzer, they identified the particle size and counted the particle number. Based on these observations, they claimed that the erosion rate in the maximum stage could be quantitatively evaluated in terms of material hardness and the fatigue crack growth rate. Recently, the authors [9] found that the morphology features of dislodged particles during the incubation period have distinctive characteristics, which differed from that for the subsequent stage.

The objective of the present study is to identify the size distribution and shape characteristics of the erosion particles and clarify their morphology features for the characteristic stages of the vibratory erosion rate-time pattern. The failure modes are also to be clarified based on particle analysis and observation of eroded surface.

\section{2- EXPERIMENTAL}

The scheme of the vibratory apparatus is shown in Fig. 1. The apparatus, except for the stationary specimen, conforms to a large extent to the ASTM standards [10] .The vibratory frequency is $19.5 \mathrm{kHz}$ and the peak-to-peak amplitude is $50 \mu \mathrm{m}$. The corresponding maximum output power is $550 \mathrm{~W}$. The horn tip is flat with $12.7 \mathrm{~mm}$ diameter and is made of SUS 304 stainless steel. The stationery specimen that was held below the horn tip has an area of $25 \times 25 \mathrm{~mm}^{2}$, which is much larger than of the horn-tip surface area, and a thickness of $2 \mathrm{~mm}$. The separation distance, $\mathrm{L}$ between the stationary specimen and the horn tip was precisely adjusted using a dial gauge and maintained at a value of $0.8 \mathrm{~mm}$. This value of distance $\mathrm{L}$ was chosen in the present work to obtain significant values of erosion rate [11].

The specimen and the end of the stepped horn were immersed in $1200 \mathrm{ml}$ open beaker, in which $700 \mathrm{ml}$ of tap water (test water) is contained. In order to stabilize the gas content and to release entrained gases, the test water was allowed to stay in an open atmosphere for at least $24 \mathrm{hr}$ [12]. Since the test water temperature markedly affects the degree of erosion and impact pressure [13], the water temperature was kept 
constant at $25 \pm 2^{\circ} \mathrm{C}$ by circulating cooling water around the beaker as shown in Fig.1. While the water temperature was the bulk temperature of the test water, this did not necessarily reflect the temperature of the water within the gap between the horn tip and specimen. It was found that for test duration of an order of minutes, the gap temperature did not exceed the bulk temperature by more than $4{ }^{\circ} \mathrm{C}$ [14]. Therefore, for decreasing the excess temperature of the gap over the controlled temperature and for less variation of gas content, the test duration time was decreased to the order of few minutes.

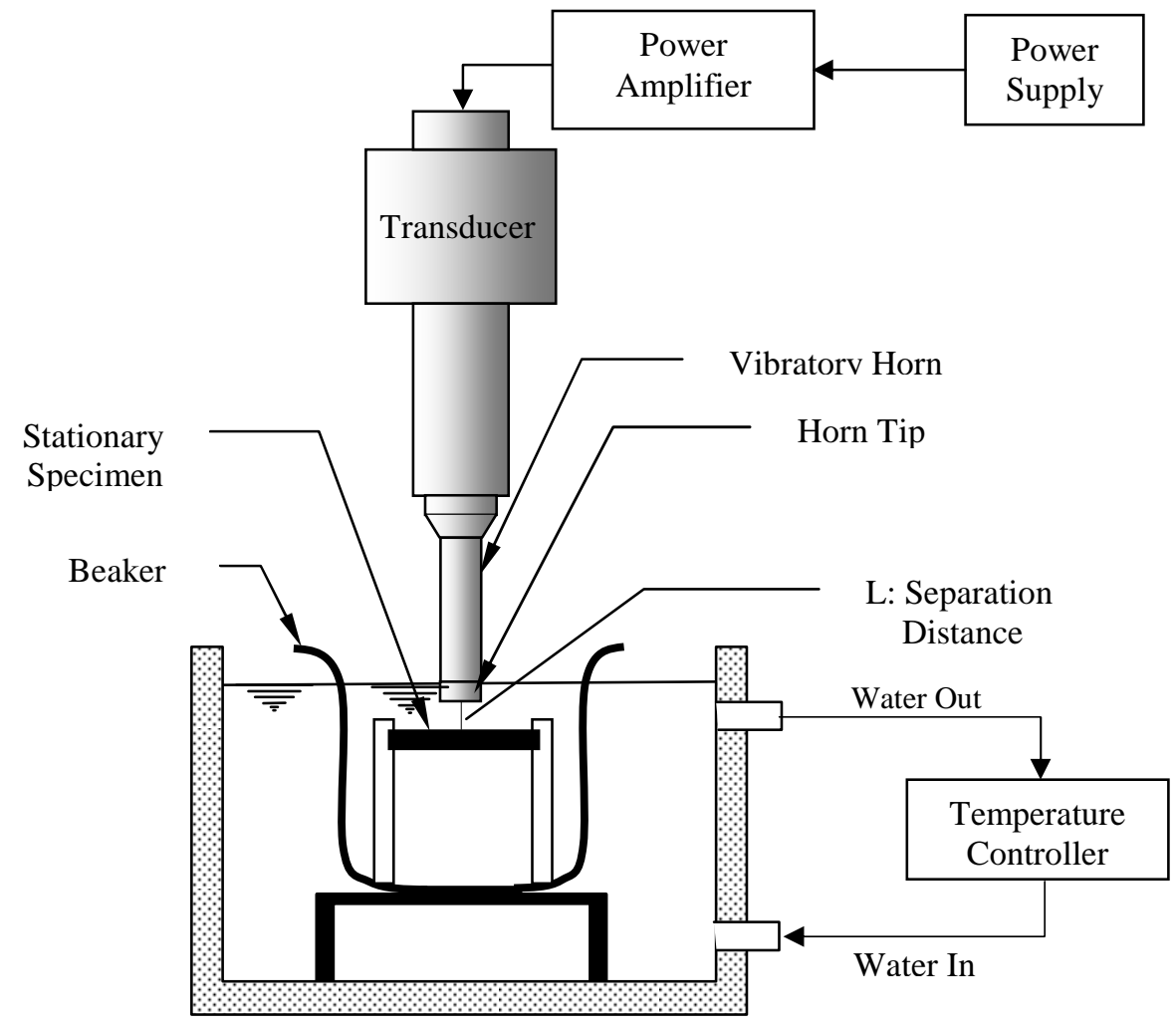

Fig. 1: Schematic view of test apparatus.

The stationary specimens were made of pure aluminum (Al-99.999) since its damage is easily detectable because of its low-wear resistance. Specimens were cut from a single block of Al-99.999 to ensure metallurgical uniformity. Since the surface roughness plays an important role in developing the erosion and the material removal [15], therefore, the specimens working faces and the horn-tip end were highly polished with grade 3000 emery paper. The resulting maximum height roughness $R_{\max }$ was 1 $\mu \mathrm{m}$ for stationary specimen and $0.025 \mu \mathrm{m}$ for the horn-tip end. Before and after each test, the specimens were rinsed in acetone and dried in air. Then specimens were weighed with a balance of $100 \mathrm{~g}$ capacity and $0.1 \mathrm{mg}$ sensitivity.

The erosion particles were collected from the test water through a plastic filter with a pore diameter of $0.45 \mu \mathrm{m}$. The filter paper was a porous cellulose nitrate membrane, which was the same as that used by Ahmed et al. [7]. The erosion particles on the filter 
were transferred to the carbon tape. Then, the morphology and chemical composition of particles were examined using scanning electron microscopy with X-ray analysis. Using the SEM image of particles and image processing software, the particles were characterized in terms of; Area (A), Equivalent diameter (d), Perimeter (P), Elongation ratio $(E L)$ and roundness factor $\left(\mathrm{P}^{2} / 4 \pi \mathrm{A}\right)$.

\section{3- RESULTS AND DISCUSSION}

\section{3-1 Time dependence of cavitation damage}

Figure 2 shows mean depth of penetration rate (MDPR) versus time. This erosion rate is composed of the following periods: (I) an incubation period; (II) an acceleration period; (III) a steady-rate period; (IV) an attenuation period. In the incubation period induces extensive plastic deformation of surface regions combined with little particle removed, the formation of pits, surface depressions and crack initiation at region of high stress and strain concentration. In the acceleration period, the erosion rate increases to the maximum level because of superficial hardening and crack development. In the maximum-rate period, the erosion rate has a constant value. This may be related to the attack on homogenous work-hardened surface. In attenuation period, the erosion rate decreases. The reduction in the damage in this period is associated with reduction of cavitation collapse pressure or cavities in the vicinity of rough surface, and cushioning effect of liquid trapped in the pockets and crevices of the eroded surface or the cushioning effect of defused air in liquid at the cavitation zone. These results are in agreement with those in [13].

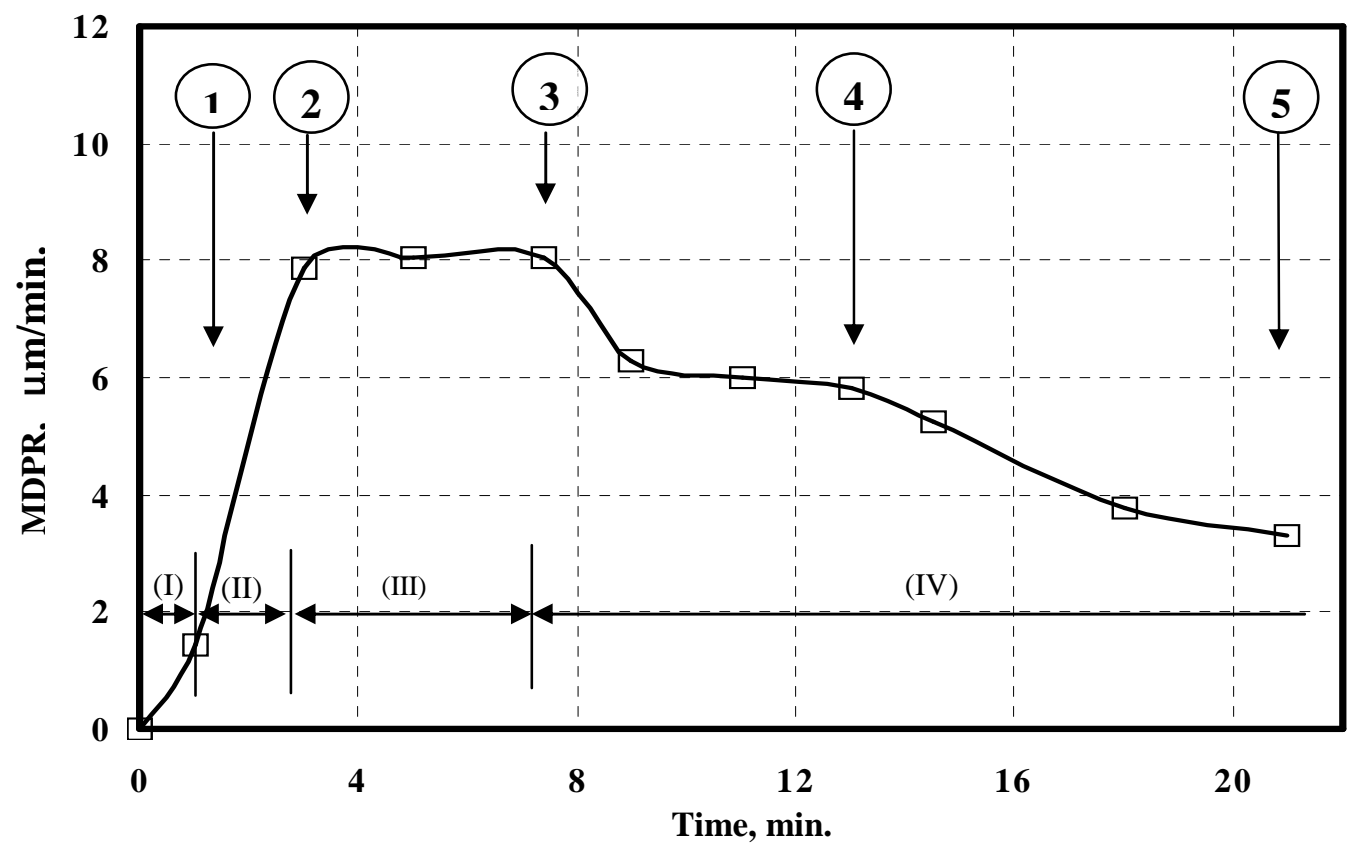

Fig. 2: Characteristic periods of the erosion-rate -time curve. 


\section{3-2 Particle characteristics}

The erosion particles were collected nearly at the end of each stage of erosion rate for the three periods; (I), (II) and (III), at points 1, 2 and 3 shown in Fig. 2. However, for the last period (IV), the particles were collected at two intervals at points 4 and 5 as shown in Fig. 2, to recognize if there is a change in the particle characteristics with erosion progress. For examining the particle morphology features, SEM photographs with high magnification were taken. For quantitative morphological analysis such as; size, perimeter, etc, a low SEM magnification images were used. All the particle characteristics were identified for each individual particle. The quantitative morphological analysis was measured for more than 100 particles at points 2-5. For point 1 , which represents the incubation period, the number of particles was few and was less than 10. Since most of particles have an irregular shape as shown in Fig. 3, the projected area diameter or equivalent diameter has been used to represent the size of the wear particles. The projected area diameter or equivalent diameter is the diameter of a circle having the same area of the projected area of the particle in same stable orientation. To characterize particle shape, ellipticity can be effectively measured and calculated. To characterize ellipticity, elongation ratio (EL) is used

$$
\mathrm{EL}=\log _{2} \text { (major axis/minor axis), }
$$

where major axis and minor axis are the axes of the Legendre ellipse [16]. The Legendre ellipse is an ellipse with the center in the object's centroid and with the same geometrical moments up to the second order as the original object area. The perimeter of wear particles was measured by employing fractal analysis.

\section{3-3 Particle morphological features}

Figure 3 shows typical particles observed for the aforementioned erosion-rate periods. As it is clear from this figure and based on the previous results [9], the particles observed during the incubation period (Photo 1) have distinctive characteristics, which differed from that for the subsequent periods (Photos 2-5). These characteristics include the lamella shape, folding and curving. In addition, one of the particle surfaces was the virgin surface. The foldness and the particles curvature were interpreted as follow: At the beginning, due to the collapse of the formed cavities the superficial layer is subjected to compressive load, while the bulk material of the specimen is not, or slightly, affected by this compressive load. This means that the superficial thin layer, which is subjected to normal compressive load, tends to elongate (spread) flatwise, but the bulk material resists this spreading. This will create residual compressive stresses in the superficial layer in the flatwise direction. This is in conformity with the residual stress measurements [17, 18] which showed that cavitation leads to the development of compressive residual stress in the sample surface. As soon as an erosion particle in the form of lamella is dislodged due to fatigue it will curve or fold depending on the intensity of the residual stresses induced in it before its removal from the specimen surface. For the particles of the last three periods (II-IV) shown in Photos 2-5, they have similar appearances. They seem to be more irregular and thicker than the particles of the incubation period. The reason for the change in the shape of particles observed in the periods II-IV from that observed in period I (i.e. incubation period) may be attributed to the following. The surface of metals is known to consist of several layers having physicochemical characteristics 
peculiar to the bulk material itself [19], Fig. 4. Since specimens are polished and rinsed in acetone before experiments, the contaminant and oxide layers are removed.
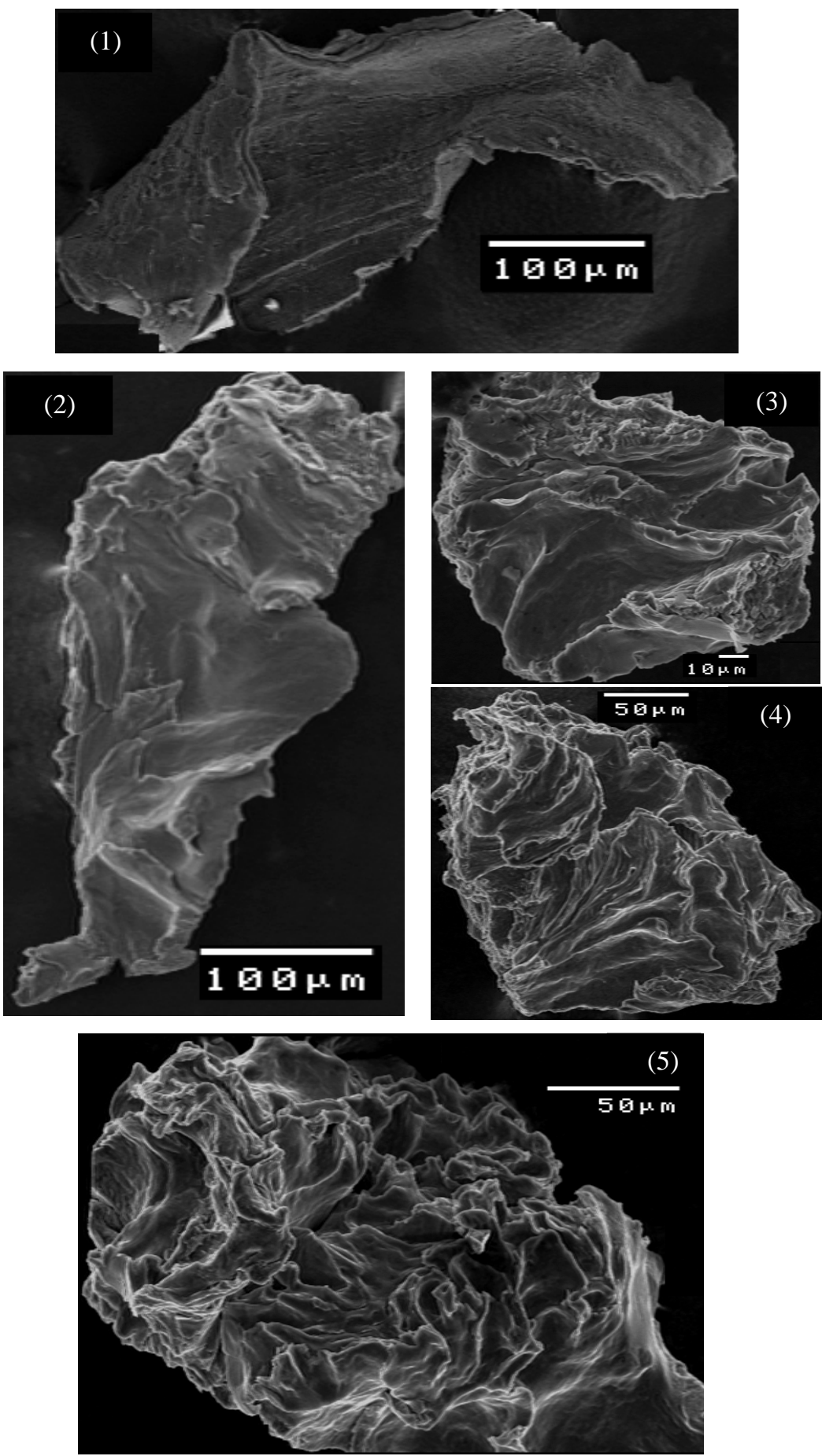

Fig. 3: Scanning electron photomicrographs of particle removed at points 1-5 shown in Fig. 2 for the erosion-rate periods. 
The specimen surface consists of the bulk material covered with thin layer of workhardened metal of thickness of about $10 \mu \mathrm{m}$. This layer is peeled of in the incubation period in the form of thin erosion particles. Now, the bulk material is subjected to cavitation erosion. Wear particles are removed from the bulk material by fatigue. Fatigue wear is characterized by the formation of large wear fragments. Therefore, wear particle in periods (II-IV) are thicker than the particles in the incubation period.

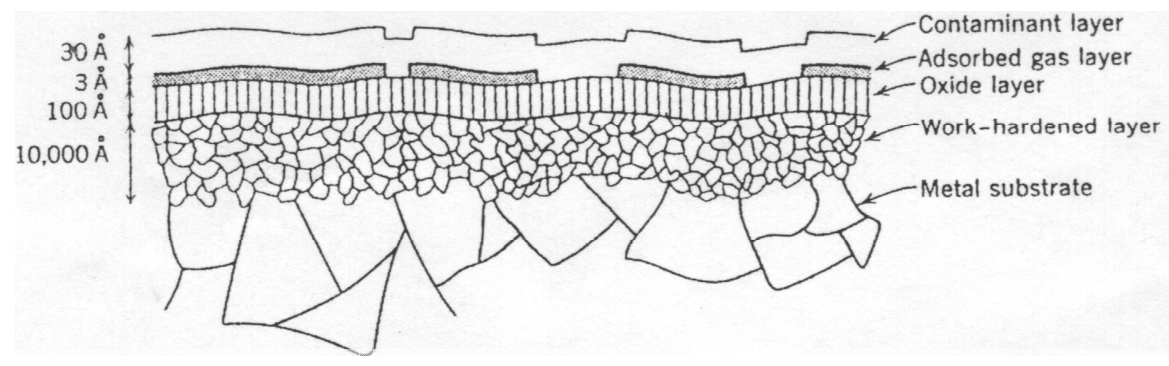

Fig. 4: Schematic illustration of films on a metal surface (not to scale).

Based upon the features of particle morphology presented here and elsewhere [9], it can be concluded that, the observation of particle morphology during incubation period can be used as a successful tool to detect early the cavitation erosion.

The topography of the detached particles is a negative replica corresponding to that of cavitation erosion surface. Therefore, surface examination of wear particles is clearly a reliable technique for predicting the cavitation erosion mechanism. It is easy to observe the crack propagation marks on the surface of all the particles presented in Fig. 3. This gives evidence that all the particles were removed by fatigue for all the erosion-rate periods. These results confirm the previous results [7, 13, 15] that the dominant mode of cavitation failure for ductile material is fatigue failure.

\section{3-4 Particle size distribution}

In the incubation period, particle sizes with diameter less than $100 \mu \mathrm{m}$ have not been observed. The particle diameter range is between 100 and $300 \mu \mathrm{m}$. The mean particle size is about $207 \mu \mathrm{m}$. Such large particles cannot be successfully described by microjet, which is resulted in pit formation of several micrometers in diameter. This is in agreement with the result reported by Ahmed et al. [15] that the important mechanism to transfer the cavitation energy to the solid is only explained by shock pressures accompanied by collapsing massive bubbles. Therefore, based on the feature morphology of particle, mentioned above, and their large sizes, it can be stated that the predominant failure mode from the early period of cavitation erosion is fatigue.

Figure 5 shows the distribution of the particle size for the last three periods; namely: acceleration, steady-rate and attenuation period. A skew symmetrical distribution to the left side appears for acceleration, steady-rate and the second interval of attenuation period. This means that the diameter of most frequently removed particles is less than $125 \mu \mathrm{m}$. However, the distribution in first interval of the attenuation period is normal and the majority of the particles have a size of about $185 \mu \mathrm{m}$. It can be seen from this figure that the maximum diameter is about $360 \mu \mathrm{m}$ in 

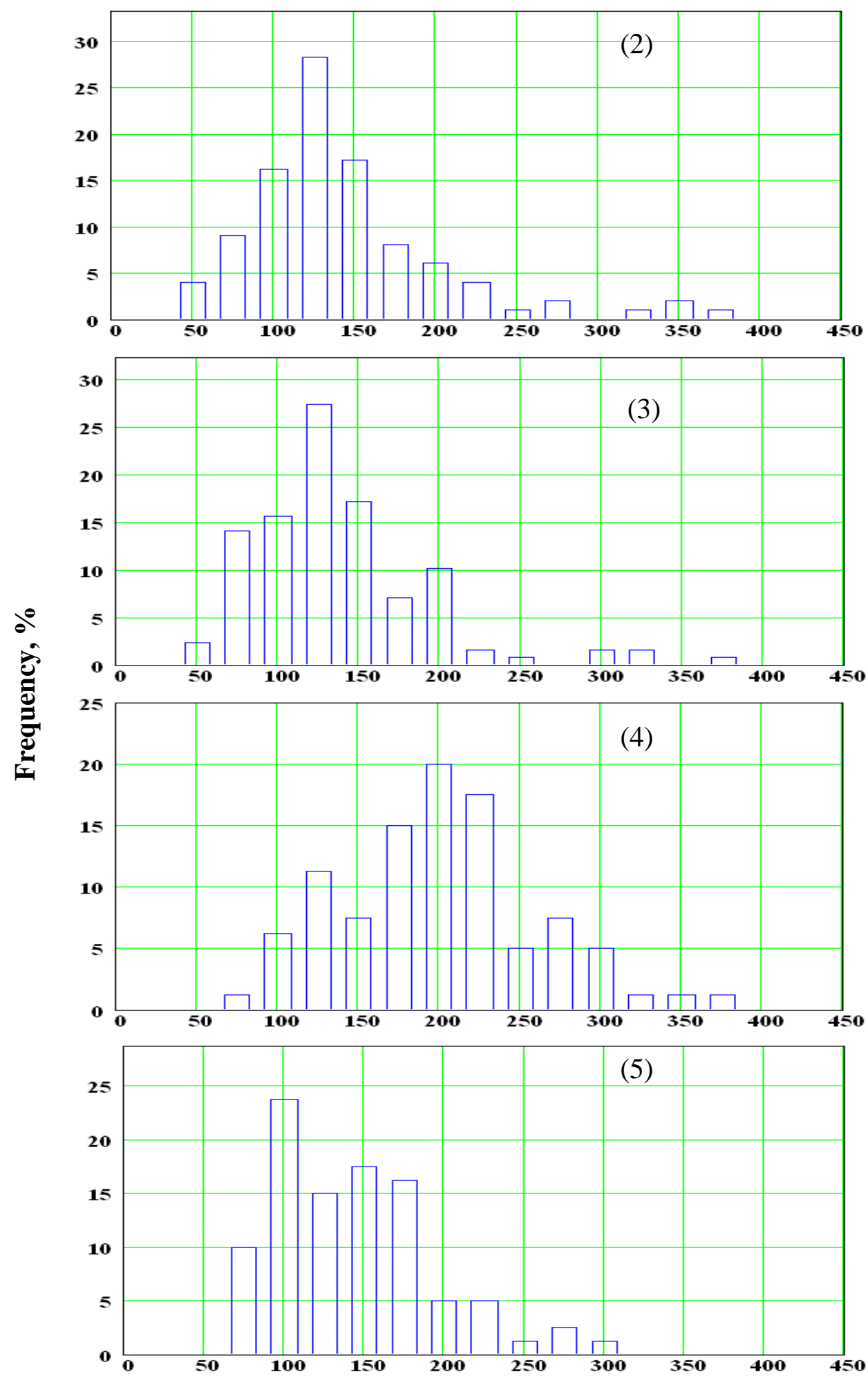

Size. um.

Fig. 5: Size distribution of particles removed at points 2-5 shown in Fig.2. 
the acceleration, steady-rate and first interval of attenuation period. Then the maximum diameter diminishes in the second interval of attenuation period to become about 300 $\mu \mathrm{m}$. It can also be observed that the particles of size less than $50 \mu \mathrm{m}$, which observed for acceleration and steady-rate period, disappeared in the attenuation period. The process of crack spreading which decreases as result of the reduction of cavitation collapse pressure and cushioning effect in this period can interpret the decrease in the size. As the process of crack spreading decreases the frequency of crack interlock decreases and this in turn will reduce the formation of small particles sub $50 \mu \mathrm{m}$. Figure 5 illustrates also that there is similarity in the distribution of particle size for acceleration and steady-rate period. This similarity is in consistence with the data shown in Fig. 2, where the MDPR for the two periods is equal.

\section{3-5 Particle shape}

Since wear particle profile, or shape characteristics, are important features that can be exploited very effectively to identify/classify particles in relation to the ongoing wear process [20], the shape factor in terms of elongation ratio (EL) and roundness factor are presented in Figs. 6 and 7. It is seen from Fig.6 that EL for the particles in incubation period is nearly twice that for the particles in the subsequent periods. This enhances the previous result that the particles in the incubation period have distinctive characteristics. EL has a value of about 1.09, i.e. the particles have an elliptical shape with the axes ratio 7:3. However, for the last three periods II-IV, the values of the elongation ratio vary in a small range of 0.59 to 0.46 . This corresponds to the ellipse with the axes ratio 7:5, i.e. approaches from spherical shape.

Figure 7 shows also that the particles in the incubation period have the largest value of roundness factor. The roundness factor is equal to unity for circle shape and any increase over unity indicates departure from circularity (or sphericity in 3-D context). Therefore, all the cavitation erosion particles in all the periods are out of circularity. It can be concluded based upon EL and RF that the particles in incubation period have the larger longitudinal shape than that for the particles in the subsequent periods.

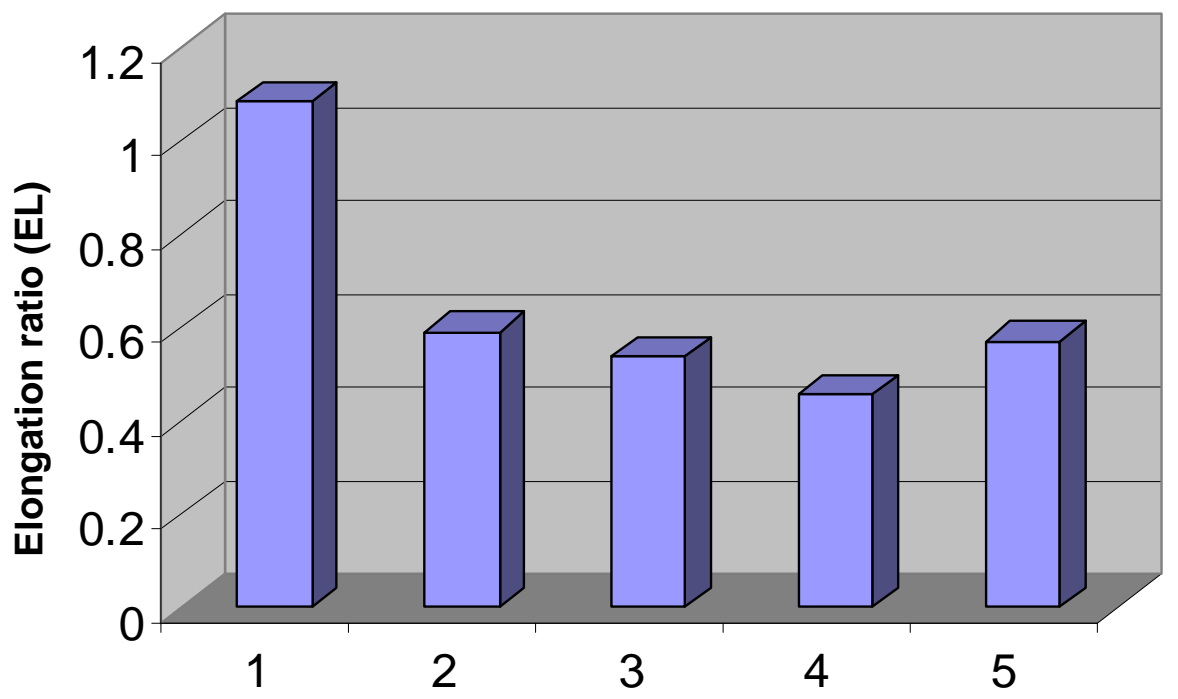

Fig. 6: Elongation ratio (EL) of particles removed at points 1-5 shown in Fig. 2. 


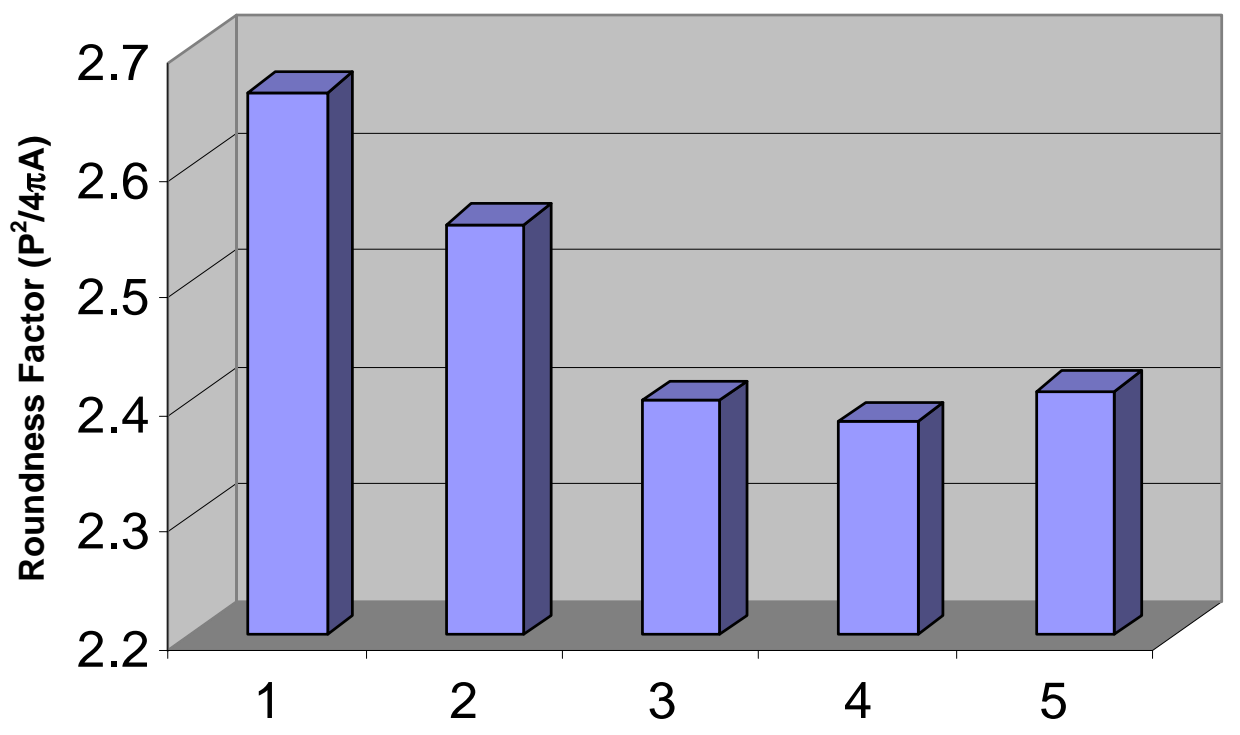

Fig. 7: Roundness factor (RF) of particles removed at points 1-5 shown in Fig. 2.

\section{4- CONCLUSIONS}

From the results and discussion presented above, the following conclusions can be drawn,

1- The particles removed during the incubation period have distinctive morphological features that differed from that for the subsequent periods. These features include the lamella shape, folding, curving and one of the particle surfaces was the virgin surface. However, the particles removed during the last three periods of erosion process have similar appearances. They have irregular shape and are thicker than that for the incubation period.

2- The particle size distribution was limited during the incubation period and was in the range of 100 to $300 \mu \mathrm{m}$. However, the size distribution was in the range of 25 to $360 \mu \mathrm{m}$ for the last three periods.

3- The particles removed during the incubation period are the most longitudinal one because these particles have the maximum value of elongation ratio (EL) and roundness factor (RF).

4- The observation of particles characteristics during the incubation period can be used as a successful tool to detect early the cavitation erosion occurrence.

5- All the particles removed in all cavitation periods were out of sphericity because the roundness factor (RF) was larger than 2.

6- The particle morphology reveals that the vibratory cavitation erosion process is due to fatigue in all periods 


\section{REFERENCES}

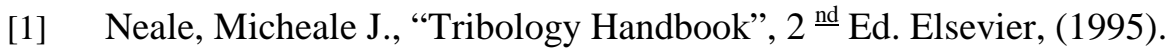

[2] Hunt, T.M., "Handbook of wear debris analysis and particle detection in liquids", Essex, England, Elsevier, (1993).

[3] Sperrin, T.P. and Nowell, T.T., "SYCLOPS- a qualitative debris classification system developed for RAF early failure detection centers", Tribology Int., pp.1-6(2005)

[4] Steller J., "International cavitation erosion test and quantitative assessment of material resistance to cavitation", Wear, 233-235, pp. 51 - 64, (1999).

[5] Kenkermath D. and Thiruvengadam A., "Analysis and characterization of particles produced by cavitation erosion", Proc. Conf. cavi., 1 Mech E, Edinburgh, pp. 285-295, (1974).

[6] Tomlinson W. J. and Chapman D., "Effect of amplitude on the erosion and corrosion of copper by $20 \mathrm{kHz}$ ultrasound", Wear, 27, pp. 381 - 384, (1974).

[7] Ahmed S. M., Hokkirigawa K., Kikuchi K., Higuchi J. and Oba R., "SEM studies of particles produced by cavitation erosion”, JSME Int. J., Ser. B , 3624, pp. $517-523$, (1993).

[8] Hattori S. and Nakao E., "Cavitation erosion mechanism and quantitative evaluation based on erosion particles", Wear 249, pp. 839 - 845, (2002).

[9] Abouel-Kasem A. and Ahmed S.M., "Cavitation erosion mechanism based on analysis of erosion particles," Journal of the Egyptian Society of Tribology (EGTRIB J.), Vol.2, No.3, pp1-15, (2004).

[10] ASTM Stand-G32-92, ASTM Stand-G32-92,in: Annual book of ASTM standards, Part 03.02, ASTM, Philadelphia, PA, pp. 97, (1995).

[11] Vyas B. and Preece C. M., "Stress produced in a solid by cavitation", J. Appl. Phys. 47 (12), pp. 5133 - 5138, (1976).

[12] Ahmed S. M., Ito Y., Higuchi J. and Oba R., "A peculiar behavior of cavitation nuclei size distributions in sample water under vibratory erosion tests", JSME Int. J., Ser. II, 33(4), pp. 629 - 633, (1990).

[13] Ahmed S. M., "Investigation of the temperature effects on induced impact pressure and cavitation erosion", Wear, 218, pp. 119 - 127, (1998).

[14] Singer B. C. and Harvey S. J., "Gas content and temperature effects in vibratory cavitation tests", Wear, 52, pp. 147-160, (1979).

[15] Ahmed S. M., Hokkirigawa K. and Oba R., "Fatigue failure of SUS 304 caused by vibratory cavitation erosion", Wear, 177, pp. 129 - 137, (1994)

[16] Mikli V., Kaerdi H., Kulu P. and Besterci M., "Characterization of powder particle morphology," Proc Estonian Acad. Sci. Eng., pp. 22-34 (2001).

[17] Krause, H. and Mathias, M., "Investigation of cavitation erosion using X-ray residual stress analysis", Wear, 119, pp.343-352(1987).

[18] Mathias, M., Gocke, A. and Pohl, M., "The residual stress, texture and surface change in steal induced by cavitation", Wear, 150, pp.11-20, (1991).

[19] Halling, J., "Principles of Tribology". Mc Millon press limited 1979

[20] Raadnui, S., "Wear particle analysis-Utilization of quantitative computer image analysis": A review, Trib. Int., pp. 1-8 (2005). 


\section{وصف الحبيبات الناتجة عن تآكل التكهف بتحليل صورها المجهرية}

الخصائص السطيّة التفصيلية للحبييات الناتجة عن تآكل التكهف سوف تكون ذاتِ فات مغزى في

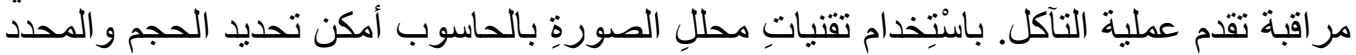

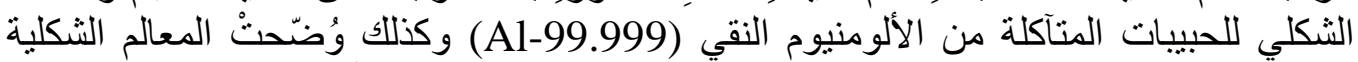

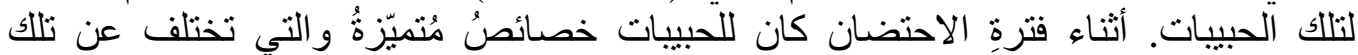

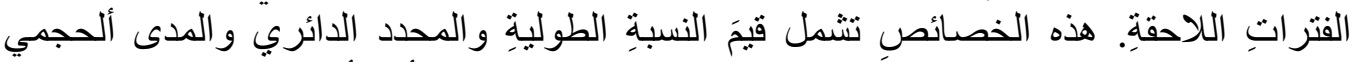

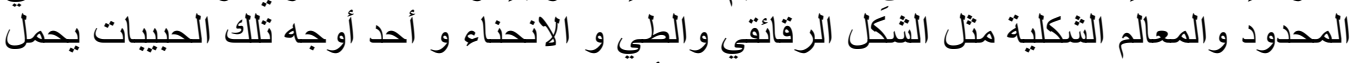

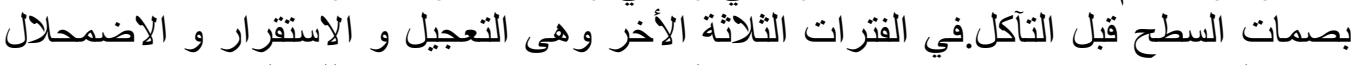

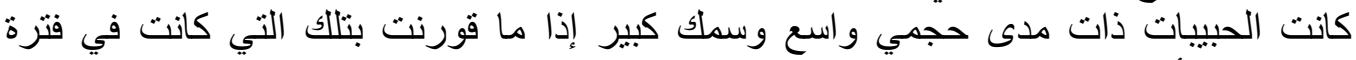

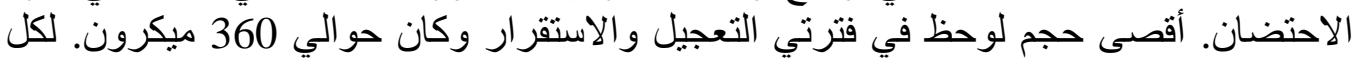

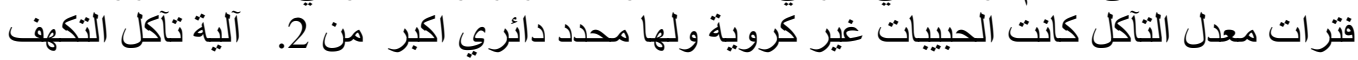

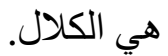

